\title{
Factores socioeconómicos que influyen en la salud nutricional y actividad física de escolares \\ Influence of socioeconomic factors in the health state of primary education students
}

\author{
Antonio Sánchez-U rrea, Tomás Izquierdo Rus \\ Universidad de Murcia (España)
}

\begin{abstract}
Resumen. Introducción. Las desigualdades socioeconómicas pueden ser una de las causas que modifiquen los patrones al imenticios y actividad física de nuestros estudiantes. La obesidad y el sobrepeso son un problema en la sociedad actual, y su tendencia ascendente, especialmente en la población infantil, han hecho que sean consideradas como la epidemia del siglo XXI según organizacionesinternacionales. Material y métodos. Esteestudio, bajo un enfoque cuantitativo, tiene como propósito describir la realidad del estado de salud de los estudiantes, por lo que se realiza un estudio descriptivo con 154 estudiantes de centros educativos de la Región de M urcia. Se anal izan los datos extraídos de los estudiantes a través de tres cuestionarios y la medición de talla y peso. Se val ora el estado nutricional mediante la medición del índice de Masa Corporal (IMC = Peso/ Altura²). La clasificación del IMC se realiza mediante las tablas establecidas por el proyecto Food and Nutrition Technical Assistance III Project, en función del género y la edad. Resultados. El sobrepeso y la obesidad parecen estar asociados con más variables aparte de la actividad físicay la alimentación, ya queéstas, se pueden encontrar afectadas por el nivel socioeconómico y cultural. Conclusiones. Cabe destacar la existenciade diferencias significativas tanto en lasituación socioeconómica como en el estado de sal ud del alumnado, lo que muestra la asociación entre la economía de la familia y la sal ud nutricional de sus hijos. El estudio final iza con implicaciones educativas y nuevas líneas de investigación sobre el tema.
\end{abstract}

Palabras clave: Condiciones socioeconómicas, Nutrición, Familia, Educación Primaria.

\begin{abstract}
Introduction. Socio-economic inequal ities can be one of the causes of the eating patterns and physical activity of student's change. 0 besity and overweight are a problem in today's society, and its trend upward, especially in children, have made that they should be consider ed as theepidemic of the 21st century according to International organizations. Material and methods. This study aims to describe the reality of the State of health of students, so it is a descriptive study with 154 students from schools in the Region of Murcia. It's analyzed the data extracted from the students through three questionnaires and measurement of height and weight. Nutritional status by measuring the rate of body mass (BMI = weight/ Altura²) is eval uated. BMI classification is performed using the tables established by the project Food and N utrition Technical Assistance III Project, gender and age. Results. O verweight and obesity appear to be associated with more variables apart from the physical activity and nutrition, since these can be affected by socio-economic and cultural level. Conclusions. It is worth noting the existence of significant differences both in the socioeconomic situation and in the state of health of the students, which shows the association between the economy of the family and the nutritional health of their children. This study ends with new lines of research on the subject and educational implications.
\end{abstract}

Key words: Socioeconomic conditions, Nutrition, Family, Physical activity, Primary education.

\section{Introducción}

\section{Estado de la cuestión}

En la actualidad, los altos índices de obesidad se han consolidado como uno de los principales problemas de la sociedad a nivel mundial (Martínez et al., 2017). En Europa la obesidad es considerada como un problema de salud pública que ha aumentado especialmente (Brettschneider, 2017).

Dicho problema de salud es denominado como la

Fecha recepción: 16-04-20. Fecha de aceptación: 09-10-20 Tomás Izquierdo Rus

tomasizq@um.es enfermedad del siglo XXI por las dimensiones adquiridas a lo largo de las últimas décadas, y por el impacto sobre la morbi-mortalidad, la calidad de vida y el gasto sanitario (Alba-Martín, 2016). Siguiendo con la misma autora, esta enfermedad se caracteriza por la acumulación excesiva de grasa o hipertrofia general del tejido adiposo en el cuerpo. Las causas de la obesidad son complicadas e incluyen factores genéticos, biológicos, del comportamiento y culturales (Alba-Martín, 2016). Básicamente, la obesidad ocurre por un desequilibrio entre el consumo y el gasto de energía.

La obesidad se ha convertido en una de las enfermedades más generalizadas en la sociedad del siglo XXI, 
con especial influencia en la población infantil (Chacón et al., 2017).

\section{La obesidad infantil}

En 2017, la OMS analizó la evolución del IMC y obesidad desde 1975 hasta 2016 mostrando que la obesidad infantil y adolescente se multiplicó por 10, pasando de 11 millones a 124 millones (O rganización M undial de la Salud, 2018). Igualmente, 213 millones de jóvenes presentaban sobrepeso sin llegar a alcanzar el umbral de obesidad. La OMS establece el inicio de la adolescencia a los 10 años (O rganización M undial de la Salud, 2018). Según esta clasificación, escolares de 5y y 60 curso de Primaria podrían incluirse dentro de esta complicada etapa, concretamente en la adolescencia temprana. En España, encontramos un 4.7\% de pobla ción adolescente que sufre obesidad, existiendo una diferencia considerable entre chicos $(6.4 \%)$ y chicas $(2.9$ $\%$ ) (Inchley, Currie, Jewell, Breda, y Barnekow, 2017).

La niñez enfrenta diferentes situaciones que influyen en su desarrollo, pero desde finales del siglo XX y durante las dos primeras décadas del siglo XXI, han ga nado importancia las problemáticas por inactividad física, el sobrepeso, la obesidad y factores de orden psicológico, como elementos de riesgo que atentan contra la salud de esta población, así como para otros grupos de edad, y según varias evidencias, estos problemas tienen orígenes multifactoriales, tanto sociales, fisiológicos, metabólicos, moleculares, como genéticos (Cigarroa, Sarqui \& Zapata-Lamana, 2016).

A nivel mundial, al año 2016, el $18 \%$ de los niños entre 5 y 18 años presentaron sobrepeso, mientras que el $6 \%$ de los niños y el $8 \%$ de las niñas presentaron al gún grado de obesidad (W HO, 2016). La prevalencia de obesidad entre niños y adolescentes se incrementó diez veces de 1975 al 2016; esto podría deberse en parte a que la urbanización ha facilitado el acceso a la comida chatarra y reducido las zonas de juego y espacios para el esparcimiento seguro (Clark, et al., 2020).

\section{Alimentación y actividad física}

La promoción y acceso inmediato al consumo de alimentos industriales baratos y ricos en calorías, junto a la escasez de métodos preventivos para este problema, la escasa información a las familias sobre dicho problema y sobre cómo ayudar a paliarlo, la falta de actuaciones directas en los centros educativos en atención para la salud adecuados a afrontar el sobrepeso y la obesidad de los escolares desde las aulas, son el principal motivo de esta enfermedad (Ríos, 2019). Dicha alimentación puede producir trastornos y enfermedades más allá del sobrepeso o la obesidad como son enfermedades de tipo cardiovascular, hipertensión, diabetes o problemas psicológicos (Esteves, Vieira, Bras, $\mathrm{O}$ 'H ara y Pinheiro, 2017).

Por otro lado, España muestra importantes estudios sobre la adherencia a la dieta mediterránea como el estudio O BIN (Naranjo-O rellana, Alonso-Alfonseca, Carranza-Márquez \& Rueda-Puente, 2018), donde se evaluaron los hábitos alimenticios a través de un estudio longitudinal entre el sexo de los menores durante los años 2011-2017. A diferencia de la tendencia observada respecto a la práctica de $A F$ y el género, tanto el estudio ENKID como el OBIN no mostraron diferencias para la alimentación en función del sexo. La dieta mediterránea a su vez, ha sido relacionada con una mayor satisfacción con la vida y con mejor bienestar mental (Rosa et al., 2019).

\section{Actividad física en la población infantil}

La actividad físicaes predominante debido a que tanto la capacidad cardiorrespiratoria como la fuerza muscular, ambos componentes de la condición física general, han demostrado influir de forma significativa en la salud de adultos (Campos, González-Víllora, Gómez, $\&$ Martins, 2019). Un buen nivel de condición física es capaz de atenuar los efectos negativos de al gunas condiciones fisiopatológicas, tales como la insulino-resistencia y el riesgo cardiometabólico (Vásquez et al., 2017).

Por desgracia, los niveles de actividad física realiza da por los adolescentes españoles son claramente inferiores a las recomendaciones (Ramos, J iménez-Iglesias, Rivera \& Moreno, 2016). La asignatura de Educación Física, dado su carácter obligatorio en el currículum educativo, es para muchos adolescentes el único lugar donde real izan actividad físicaregularmente (Sevil, A bós, Generelo, Aibar \& García-González, 2016). Por lo que, España destaca por ser uno de los países de Europa con mayor sedentarismo, así como un mayor sobrepeso entre la población infantil (Rosa, Carrillo, García, Pérez, Tarraga \& Tarraga, 2019).

\section{Factores económicos y actividad física}

En estudios como el Doepke \& Zilibotti (2019) se observan que hay una relación entre los hábitos alimenticios con laactividad físicay losfactores socioeconómicos del alumnado, por lo tanto, en este estudio se analizan los hábitos de práctica físico-deportiva y de alimenta ción delosescolares, junto alos aspectossocioeconómicos que influyen en la práctica físico-deportiva y alimenta 
ción.

La OMS (2012) informó que los factores económicos influyen de forma directa en la actividad física y la salud de los discentes, la estrategia mundial orienta a los países a desarrollar estilos de vida saludables, centrándose en parte en los factores socioeconómicos entre otros. Según diferentes estudios, los factores más importantes que condicionan la aparición de sobrepeso y obesidad son el sexo, el nivel de educación de los progenitores/ cuidadores, el nivel de ingresos y los elementos relacionados con los estilos de vida, como desayunar a diario, dormir las horas de sueño recomendadas, el nivel diario de actividad física intensa y la percepción del cuidador del estado ponderal de los niños. (AjejasBazán, Soto, Del Carmen, Vázquez-Sellán, DíazMartínez \& Domínguez-Fernández, 2018).

\section{Enfermedades consecuentes por la obesidad}

La obesidad es el trastorno nutricional más frecuente en la adolescencia; asociado a numerosas comorbilidades tales como la hiperinsulinemia y la resistencia a la insulina; ambas son el eje central del desa rrollo posterior de estados de intolerancia a la glucosa, diabetes tipo 2 y/ 0 síndrome metabólico (PalominoDevia, O tero-Saborido, \& González-Jurado, 2016). La excesiva ingesta calórica y la disminución de la actividad física han generado un aumento sistemático de diversas variables de la composición corporal en niños y adolescentes, traduciéndose en un mayor riesgo de sufrir enfermedad cardiovascular en la adultez (W HO, 2016). Estos datos, combinados con unaadiposidad y baja condición física æeróbica van generando problemas relacionados con enfermedades cardiovasculares (Latorre, Mora \& García, 2016).

El funcionamiento familiar y el autoconcepto pueden afectar los niveles de ansiedad y autoestima de adolescentes con obesidad, lo que en general incide en los efectos del tratamiento para control del peso corporal (Taylor, et al., 2017). A su vez, la prevalencia de estudiantes con problemas en su desarrollo motor es cada vez mayor, asociándose a parámetros de salud mental como unamenor autoestima, un peor autoconcepto, una baja percepción de competencia atlética, sufrir de ansiedad social y acoso escolar (Carrillo-López, 2018). Pero, además, el peso excesivo en menores se ha asociado con trastornos psicosociales, en especial con problemas en autoestima, así como con un bajo desempeño físico en esta población (ver, por ejemplo, los resultados del estudio de Delgado-Floody, Caamaño-Navar rete, JerezMayorga et al, 2018, realizado en escolares chilenos).
La baja autoestima en los menores es cada vez más común, en una sociedad donde la identidad personal depende directamente de los estereotipos que dicta la sociedad (D elgado-Floody, Caamaño, 0 sorio et al. , 2017), y la obesidad se asocia con una disminución en la memoria, funciones ejecutivas y velocidad de procesamiento (Deckers, Van Boxtel, Verhey \& Köhler, 2017).

\section{Propuestas para paliar las enfermedades}

Actualmente, se ha descrito que el $81 \%$ de adolescentes con edades entre 11 y 17 años no cumplieron las recomendaciones dictadas por la O MS en 2010, siendo las chicas menos activas (84 \%) que los chicos (78\%) (O rganización M undial de laSalud, 2018). En laactualidad, se ha consensuado la necesidad de unamejoratanto de las pautas de práctica de AF como de alimentación a nivel mundial (Afshin et al., 2019). Una intervención educativa nutricional basada en la teoría social cognitiva ayudó a mejorar los hábitos dietéticos y algunos aspectos psicológicos en mujeres (Bagherniya, et al., 2017).

A su vez, en dicho informe se confirma que la práctica de actividad física genera importantes mejoras para la salud de quien la real iza, sobre todo en lo que respecta a nivel físico y psicológico (M uros, Cofre, Arriscado, Zurita y Knox, 2017). Algunos de los efectos descritos hacen referencia a la reducción del riesgo de padecer alguna enfermedad cardiovascular, diabetes o cáncer. Igualmente, en Andalucía se comprobó que existe una relación positiva entre unas buenas pautas alimentarias y la práctica deAF con mejoras cognitivas en adolescentes (Knox y Muros, 2017).

Algunos de los principales beneficios de la AF en adolescentes han sido referidos a la mejora de la salud cardiovascular, mejorando factores determinantes como la presión sanguínea (Son, Sung, Bharath, Choi, y Park, 2017), la reducción de hasta un $40 \%$ del riesgo de sufrir cáncer asociado a enfermedades crónicas (Iglesias, Del Pozo y López, 2019). Existen también evidencias científicas que remarcan los beneficios de la práctica de AF por provocar mejoras considerables en los procesos cognitivos, además de los procesos de memorización y atención de losadolescentes (Guillamón, Canto y López, 2019). La práctica de actividad física demuestra ser beneficiosa a nivel fisiológico, psicológico y social (Sanz, 2017). Además, la actividad física en la sociedad actual es un tema elemental y prioritario, siendo de especial relevancia en las políticas sociales y de salud (Zurita, Castro, Linares y Chacón, 2017).

La salud y la calidad de vida en los menores está asociada a la práctica de la actividad física (Poitras et al., 
2016), reduciendo el tiempo dedicado a las pantallas y al sedentarismo (Saunders y Vallance, 2017).

\section{Consideraciones finales}

El interés por la obesidad infantil se ha acrecentado en los últimos años en todos los países desarrollados. Suscita gran inquietud el que su prevalencia haya aumentado a lo largo de los últimos 20 años entre dos y tres veces (Alba-M artín, 2016). Es fundamental ser críticos con la sociedad actual, una sociedad influenciada por políticas que fomentan unos estilos de vida que perjudican la salud. Transmitir un conocimiento a la comunidad educativa sobre las causas de este problema para desarrollar las estrategias que lo disipen y evitar las consecuencias que pueden suponer para la salud del escolar son metas a conseguir en esta investigación.

Los medios de comunicación han tendido a presentar la obesidad en general, y la infantil en particular, como un problema relacionado con un comportamiento irresponsable por parte del individuo, por lo que el papel de los gobiernos y de la regulación sería limitado (Atanasova \& Koteyko, 2017; Van H ooft, Patterson, Lof, Alexandrou, Hilton \& N imegeer, 2018). La prevención requiere una educación en cuanto a alimentación y actividad física, junto a la lucha contra la información masiva de los medios de comunicación de modo que adquieran hábitos saludables.

Con el conocimiento de los hábitos alimenticios, actividad física y factores socioeconómicos que rodean al discente, así como las actividades específicas desarroIladas en cada centro, el docente de Educación Física puede planificar sus sesiones orientadas a las necesidades físicas de los escolares y aportar información eficaz sobre la alimentación y la actividad física saludable. En consecuencia, en este esudio se plantea dar respuesta a la siguiente pregunta de investigación; ¿Son determinantes los factores socioeconómicos en la salud de los estudiantes de Educación Primaria?

\section{M étodo}

\section{Objetivos}

Con los antecedentes teóricos del estudio, la presente investigación persigue el siguiente objetivo general: determinar los factores socioeconómicos que influyen en el estado nutricional de los estudiantes de Educación Primaria de la Región de Murcia. A su vez, de éste se desprenden los siguientes objetivos específicos:

1. Conocer el entorno socioeconómico del alumnado y su influencia en la salud nutricional.
2. Valorar los hábitos alimenticios de los estudiantes de educación primaria en la Región de Murcia en función del tipo de centro.

3. Analizar los niveles de actividad física de los participantes.

4. Determinar la relación entre el entorno socioeconómico, los hábitos alimenticios y la actividad física que influyen en el estado nutricional de los estudiantes.

\section{Participantes y contexto}

Participaron 154 estudiantes de centros educativos de la Región de Murcia, pertenecientes a los cursos de 40, 50 y 60 de Educación Primaria. El proceso de selección, de carácter incidental y no probabilístico, tuvo en cuenta los diferentes contextos sociales en los que se encontraban situados los centros, ya que, los centros públicos se situaban en una localización con un nivel socioeconómico medio-bajo y los centros concertados en un entorno con un nivel socioeconómico medio-alto. En su mayoría, los padres de los escolares de los centros públicos tenían un nivel socioeconómico medio por el nivel bajo de las madres y, por otro lado, los padres de los escolares de los centros concertados se situaban en el nivel alto, al igual que las madres.

\section{Instrumentos de recogida de datos}

Parala presente investigación se utilizaron tres cuestionarios, uno para conocer el entorno socioeconómico de los estudiantes, validado por diferentes profesionales de la educación y ciencias de la actividad física y el deporte, otro para conocer la actividad física que realiza ban semanalmente los estudiantes (IPAC-C) y, por último, otro para conocer la alimentación que los sujetos llevaban a cabo a lo largo de la semana (KRECE-PLUS). El cuestionario con el que se analizó el entorno socioeconómico de los escolares se compone de 11 preguntas sobre el nivel de estudios, si trabajaba o no y a qué profesión del padre/ madre/ tutor se dedicaba y la estructura familiar que tenía. Se agrupa en tres niveles según las ocupaciones desempeñadas por los progenitores: nivel económico bajo, nivel económico medio y nivel económico alto. Además, establece tres niveles, según la categoría académica: nivel cultural bajo, nivel cultural medio y nivel cultural alto. Por otro lado, el cuestionario KRECE-PLUS se compone de 14 preguntas relacionadas con el tipo de comida que injerían dia riamente, con una valoración global de 0 a 10 puntos según la siguiente clasificación: 3 (nivel nutricional muy bajo), 4-7 (nivel nutricional medio) y 8 (nivel nutricional 
alto). Por último, el cuestionario IPAQ -C compuesto por 16 preguntas sobre el tipo de actividad que realizaban en los últimos siete días, siete preguntas sobre el tiempo que estaban activos en diferentes contextos y una pregunta sobre la cantidad de tiempo que veían la televisión, jugaban a videojuegos, estaban con el ordenador etc. El resultado global de este cuestionario es una puntuación que oscila de 1 a 5 , de tal maneraque las puntuaciones más altas indican mayor nivel de actividad. Antes de iniciar los cuestionarios se explicó la forma de hacerlo, existen tres tipos de preguntas, en las tres se respondían escribiendo una «X» en la casilla de la respuesta correspondiente, en otro tipo de pregunta el escolar elige una de cuatro variables, en otra se escribía la «X» en la casillla de «SI» 0 «NO 0 » y, por último, se escribía «X» en la casilla que hacía referencia al tiempo que estuvo realizando la actividad.

En segundo lugar, para la valoración del estado nutricional, a través de la medición del Índice de M asa Corporal (IMC), se midió la talla en metros y el peso en kilogramos con un estadímetro compuesto por una báscula, utilizada para obtener el peso corporal. Y para obtener la talla, el estadímetro contenía una tabla rectangular situada perpendicularmente al suelo, sobre la cual se deslizaba en sentido vertical una pequeña tabla horizontal que contactaba con la parte superior de la cabeza. La fórmula empleada para la medición del IMC fue la siguiente: Peso $(\mathrm{Kg}) /$ Estatura $\left(\mathrm{m}^{2}\right)$, determinando los siguientes estados: normopeso, sobrepeso u obesidad. Posteriormente, se clasificó a los participantes en función de las tablas establecidas por el proyecto Food and NutritionTechnical Assstance III Project, en función del género y la edad. Se seleccionó este proyecto al ser uno de los pioneros sobre el importante aumento de la prevalencia de la obesidad infantil en España.

Por último, en este apartado, se describen las varia bles que han formado parte de esta investigación:

- Nivel socieconómico de los progenitores: variable que recoge y analiza la capacidad económica de las familias en relación con la posibilidad de que sus hijos lleven a cabo una dieta equilibrada y una actividad física completa.

- Nivel cultural de los progenitores: variable que analiza el nivel académico de los progenitores en rela ción a la capacidad de comprensión y entendimiento de los hábitos saludables de sus hijos e hijas.

- Activo en Educación Física: variable que refleja si la actividad física, durante las sesiones de Educación Física, son óptimas o no.

- Activo en el recreo: variable que contempla la situación donde los estudiantes deben alimentarse y activarse tras las sesiones de clase, dependiendo de las ofertas de juego propuestas por el centro.

- Deporte tras el colegio: variable que determina la situación deportiva de los estudiantes fuera del horario escolar, reflejando si llevan una rutina sedentaria 0 activa.

- Tiempo para ver latelevisión: variable que muestra el periodo de tiempo que los estudiantes pasan sentados frente al televisor, ordenador, teléfono móvil, etc., reflejando el sedentarismo.

- Índice de masa corporal (IMC): Variable que determina si el estado de salud de los estudiantes es saludable 0 no.

\section{Procedimiento}

Para poder realizar el estudio sobre la influencia de los factores socioeconómicos en el estado nutricional de los estudiantes, tanto el centro como las familias de los participantes fueron informados mediante un protocolo donde se describía el estudio y el procedimiento de la investigación, cumplimentaron y aceptaron el consentimiento donde aparece la información y el proceso que se iba allevar a cabo en el estudio. En dicho protocolo se informó alosparticipantes del grado de confidencial idad de los cuestionarios y el carácter anónimo de sus respuestas, así como la participación voluntaria en la investigación. Como criterios de inclusión setuvo en cuenta que los participantes formaran parte de los cursos 4 ${ }^{\circ}$, 50 y 60 de Educación Primaria y no padecieran limitaciones cognitivas o mentales.

Una vez obtenida la autorización de familiares y de los colegios de la Región de Murcia, se procedió a la cumplimentación de los instrumentos. Para ello se elaboró un informe para solicitar el material que se le entrega a la Escuela de Enfermería de Cartagena, la cual prestó para el estudio un estadímetro con lo que se realiza la toma de peso y altura de los 154 alumnos. La recogida de datos, realizada en colaboración con profesorado del área en Ciencias de la Actividad Física y el Deporte, se realizó durante el curso académico 2017/ 2018.

Antes de iniciar la medición de peso y altura de los estudiantes, se repartió el cuestionario, compuesto por tres bloques; el entorno socioeconómico, actividad física y alimentación. Para la medición se anotó el peso en kilogramos y la estatura en metros con el uso del estadímetro. Los escolares fueron examinados en pantalón corto, camisetas de mangas cortas y sin zapatillas, para tomar así su peso y altura de la forma más próxima 
y efectiva. Todas las mediciones se tomaron en una sala acondicionada para tal fin, a la misma hora del día y bajo la misma temperatura ambiente $\left(24^{\circ} \mathrm{C}\right)$. Para obtener los datos del IMC se analizaron los datos extraídos tras la medición del peso y la atura de los escolares, se utilizó la fórmula estándar; Peso (Kg)/ Altura $\left(\mathrm{m}^{2}\right)$. U na vez analizada se utilizaron las tablas establecidas por el proyecto Food and N utritionTechnicalAssistancelll Project, en función del género y la edad para su categorización. Tras la toma de datos se realizó un análisis cuantitativo, caso-control, se aplicaron los cuestionarios KRECEPLUS e IPAQ -C relacionándolos con el entorno socioeconómico.

El resultado de los cuestionarios ofreció la informa ción necesaria para conocer la utilización del tiempo libre del al umnado, la alimentación que llevaban a cabo y el entorno socioeconómico. Una vez obtenido los resultados de los cuestionarios y las mediciones de altura y peso se utilizó el programa Excel para el vaciado de los datos y, posteriormente, se utilizó el programa IBM SPSS Statistics 25 paraW indows. Los datos extraídos de los cuestionarios se relacionaron con los datos obtenidos tras el cálculo del IMC de los escolares, de esta manera se observó si el entorno socioeconómico estaba relacionado o no con el IMC de los estudiantes. Tras analizar todos los datos y adquirir las tablas que muestran los resultados, se procedió a la comunicación de las deducciones a los diferentes centros, aprovechando de esta manera para desarrollar una charla en clase con la finalidad de explicar los resultados del estudio, exponiendo el significado de cada ítem utilizado, qué causas y consecuencias provocan en su salud para que de ésta manera se intente concienciar de la importancia de la actividad física y una buena alimentación diaria para su salud.

\section{Análisis de datos e interpretación de resul- tados}

Se tabularon los datos obtenidos en los diferentes centros, para analizarlos y compararlos mediante esta dísticos descriptivos apoyados de tablas personalizadas donde se extrajeron el recuento de cada parámetro. Por objetivos, se muestran estadísticos descriptivos, a tra vés de frecuencias, y la prueba chi cuadrado para determinar la independencia entre las variables del estudio. Todos los cálculos del estudio se realizaron con los programas Microsoft Excel 2010 y el software IBM SPSS Statistics en la versión 25. El valor de significación estadística establecido fue $p<.05$. A continuación, se mues- tran los resultados de la investigación en función de los objetivos del estudio.

\section{Objetivo 1. Conocer el entorno socioeconómico} del alumnado y su influencia en la salud nutricional

Los resultados referidos al análisis descriptivo sobre el nivel socioeconómico de las familias de los diferentes centros del estudio, como se muestra en la tabla 1, se observan las diferencias significativas en las variables de nivel socioeconómico de las familias de los discentes en función del tipo de centro. En la tabla se puede observar como en los centros concertados las familias poseían mayor nivel socioeconómico junto al académico, en cambio en los centros públicos se obtuvo que las familias presentaban un nivel socioeconómico y académico más bajo que en el centro concertado.

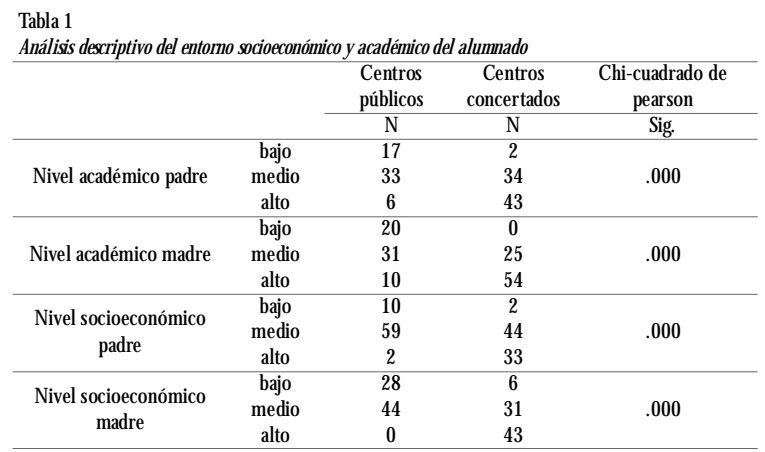

\section{Objetivo 2.Valorar los hábitos alimenticios de los estudiantes}

En la tabla 2 se puede observar cómo solamente se encuentran diferencias significativas en el ítem «idesa yunas lácteos?», dónde el alumnado del centro concertado consumía más lácteos en el desayuno que los esco-

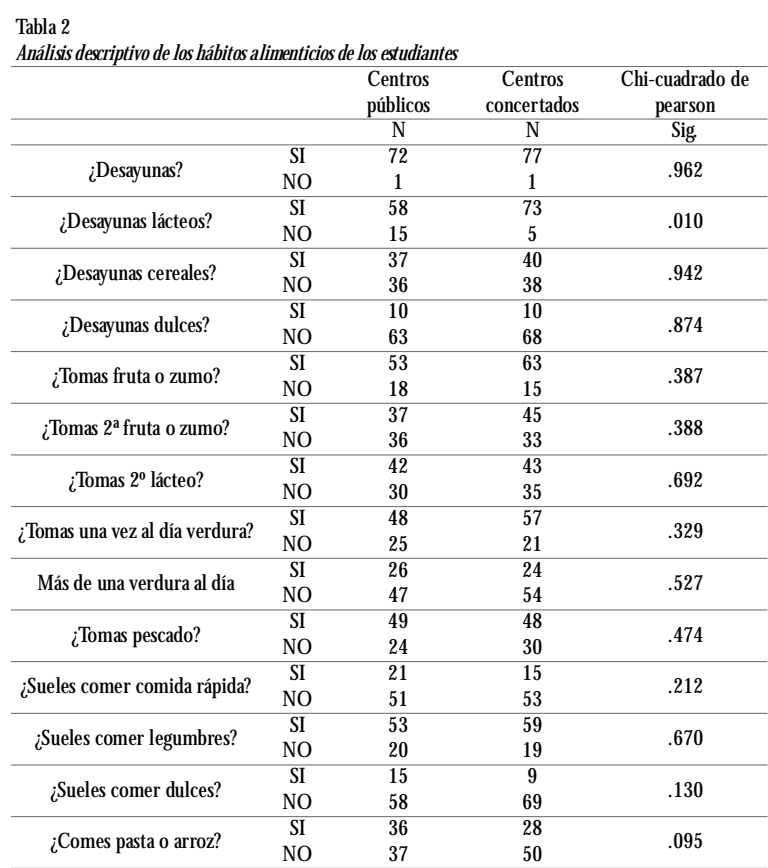


lares del centro público. El resto de ítems de hábitos alimenticios no muestran diferencias significativas entre el alumnado de los diferentes colegios. Cabe desta car que sería determinante conocer qué carne, pescado, fruta y verdura injieren en cuanto a calidad del alimento.

Objetivo 3. Analizar y comparar los niveles de actividad física de los participantes

En la tabla 3 se muestra que hay diferencias significativas en al gunos tipos de práctica físico deportiva en-

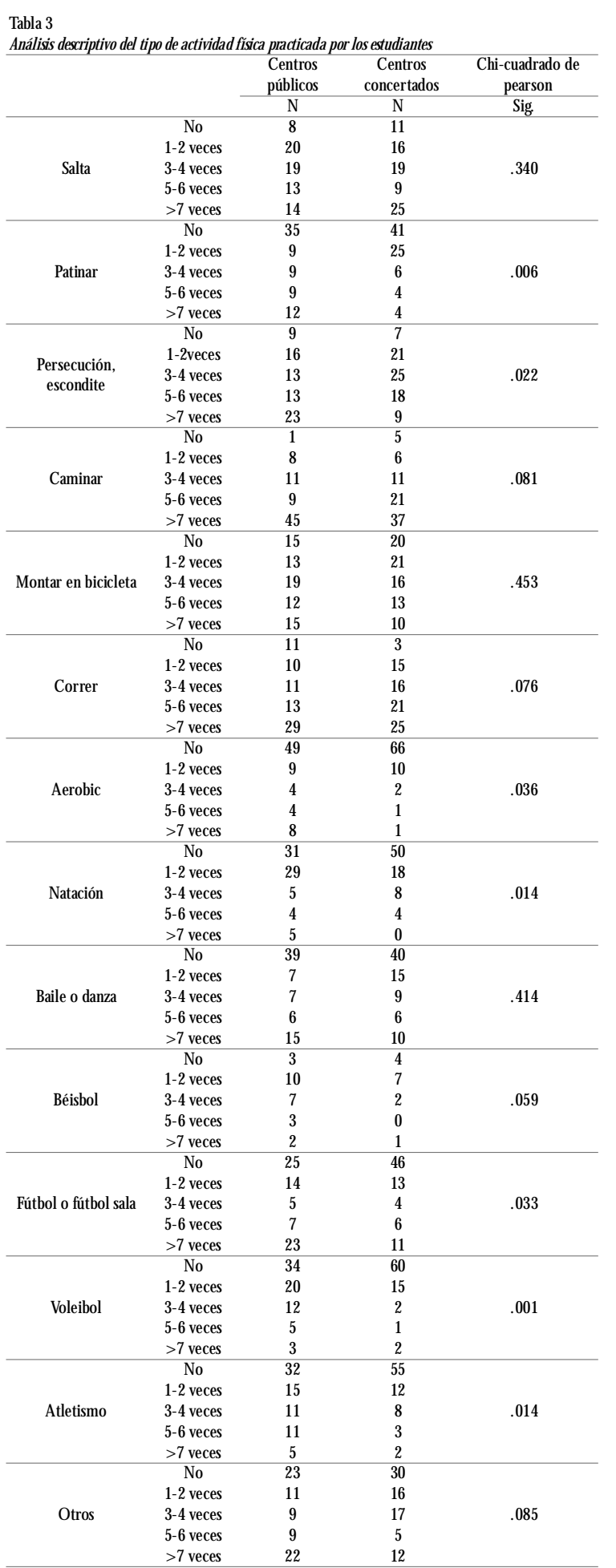

tre los diferentes centros. Destaca la diferencia significativa en la práctica de voleibol, atletismo, fútbol o fútbol sala, natación y aeróbic, dónde el alumnado del centro público lo practicaba con mayor frecuencia que el del centro concertado, mientras que juegos de persecución y patinar lo practicaban más los escolares del centro concertado.

Por otro lado, encontramos muy lejana la diferencia significativa en actividades como saltar, caminar, montar en bicicleta, correr y bailar.

En la tabla 4, se observa como existen diferencias significativas en los ítems "activo en el recreo», que puede ser debido a que la oferta de actividad del centro concertado fuese mayor que la del público, y el «deporte tras el colegio» siendo íntimamente relacionada con la actividad anterior, donde la del centro concertado superaba en actividad física al público.

Por otro lado, no se encuentran diferencias significa tivas en "activo en EF», sumando así la necesidad de más horas lectivas de EF debido a que son las horas en las que el estudiante estaba más activo. En los ítems restantes; «deporte por la noche», «deporte en el fin de semana», «tiempo para ver la televisión» y «la actividad desarrollada en los últimos siete días», no se encuentran diferencias significativas.

\begin{tabular}{|c|c|c|c|c|c|}
\hline & & $\begin{array}{l}\text { Centro } \\
\text { públicos }\end{array}$ & $\begin{array}{c}\text { Centro } \\
\text { concertados }\end{array}$ & Total & $\begin{array}{c}\text { Chi- } \\
\text { cuadrado } \\
\text { de pearson }\end{array}$ \\
\hline & & $\mathrm{N}$ & $\mathrm{N}$ & $\%$ & Sig. \\
\hline \multirow{5}{*}{$\begin{array}{c}\text { Activo en } \\
\text { EF }\end{array}$} & No hice ef & 2 & 4 & 3.90 & \multirow{5}{*}{.074} \\
\hline & Casi nunca & 2 & 1 & 1.95 & \\
\hline & Algunas veces & 5 & 17 & 14.29 & \\
\hline & A menudo & 25 & 27 & 33.77 & \\
\hline & Siempre & 40 & 31 & 46.10 & \\
\hline \multirow{4}{*}{$\begin{array}{l}\text { Activo en } \\
\text { el recreo }\end{array}$} & Estar sentado, hacer deberes, leer... & 7 & 1 & 5.19 & \multirow{4}{*}{.027} \\
\hline & Pasear por el patio & 11 & 24 & 22.73 & \\
\hline & Correr o jugar un poco & 15 & 16 & 20.13 & \\
\hline & Correr o jugar la mayor parte del tiempo & 41 & 39 & 51.95 & \\
\hline \multirow{5}{*}{$\begin{array}{l}\text { Deporte } \\
\text { tras el } \\
\text { colegio }\end{array}$} & Ninguno & 2 & 4 & 3.90 & \multirow{5}{*}{.042} \\
\hline & 1 vez en la última semana & 5 & 9 & 9.09 & \\
\hline & $2-3$ veces en la última semana & 16 & 23 & 25.32 & \\
\hline & 4 veces en la última semana & 19 & 28 & 30.52 & \\
\hline & 5 veces o más en la última semana & 32 & 16 & 31.17 & \\
\hline \multirow{5}{*}{$\begin{array}{l}\text { Deporte } \\
\text { por la } \\
\text { noche }\end{array}$} & Ninguno & 14 & 24 & 24.84 & \multirow{5}{*}{.273} \\
\hline & 1 vez en la última semana & 12 & 9 & 13.73 & \\
\hline & $2-3$ veces en la última semana & 18 & 24 & 27.45 & \\
\hline & 4-5 veces en la última semana & 18 & 12 & 19.61 & \\
\hline & 6-7 veces en la última semana & 12 & 10 & 14.38 & \\
\hline \multirow{5}{*}{$\begin{array}{l}\text { Deporte en } \\
\text { el fin de } \\
\text { semana }\end{array}$} & Ninguno & 5 & 4 & 5.84 & \multirow{5}{*}{.239} \\
\hline & 1-2 veces en la última semana & 14 & 22 & 23.38 & \\
\hline & 3-4 veces en la última semana & 20 & 27 & 30.52 & \\
\hline & 5-6 veces en la última semana & 11 & 13 & 15.58 & \\
\hline & Más de 6 veces en la última semana & 24 & 14 & 24.68 & \\
\hline \multirow{4}{*}{$\begin{array}{l}\text { Tiempo } \\
\text { para ver la } \\
\text { televisión }\end{array}$} & Menos de una hora diaria & 31 & 41 & 47.06 & \multirow{4}{*}{.142} \\
\hline & $1-2$ horas diarias & 28 & 29 & 37.25 & \\
\hline & 2-4 horas diarias & 11 & 9 & 13.07 & \\
\hline & Más de 6 horas diarias & 4 & 0 & 2.61 & \\
\hline \multirow{8}{*}{$\begin{array}{l}\text { Describe } \\
\text { tus últimos } \\
\text { siete días }\end{array}$} & Todo o la mayoría de mi tiempo libre lo & & & & \multirow{8}{*}{.696} \\
\hline & dediqué a actividades que requerían poco & 1 & 4 & 3.37 & \\
\hline & 102 veces en la última semana hice & & & & \\
\hline & actividades físicas en mi tiempo libre, & 16 & 15 & 20.26 & \\
\hline & $\begin{array}{l}\text { como correr, nadar, montar en bici etc. } \\
304 \text { veces en la última semana hice }\end{array}$ & 25 & 29 & & \\
\hline & actividad física en mi tiempo libre. & 25 & 29 & & \\
\hline & $\begin{array}{l}506 \text { veces en la última semana hice } \\
\text { actividad física en mi tiempo libre. }\end{array}$ & 19 & 22 & 26.80 & \\
\hline & $\begin{array}{l}7 \text { o más veces en la última semana hice } \\
\text { actividad física en mi tiempo libre. }\end{array}$ & 12 & 10 & 14.38 & \\
\hline
\end{tabular}


En la tabla 5 no se encuentran diferencias significativas entre el alumnado de un centro y otro en la práctica de la actividad física semanal que realizaban, estando relacionada con el último ítem de la tabla 4; «describe tus últimos siete días». En esta tabla se muestran los datos recogidos en relación a la práctica físico-deportiva de los escolares en cuanto al nivel de actividad física que desarrollaban a lo largo de la semana.

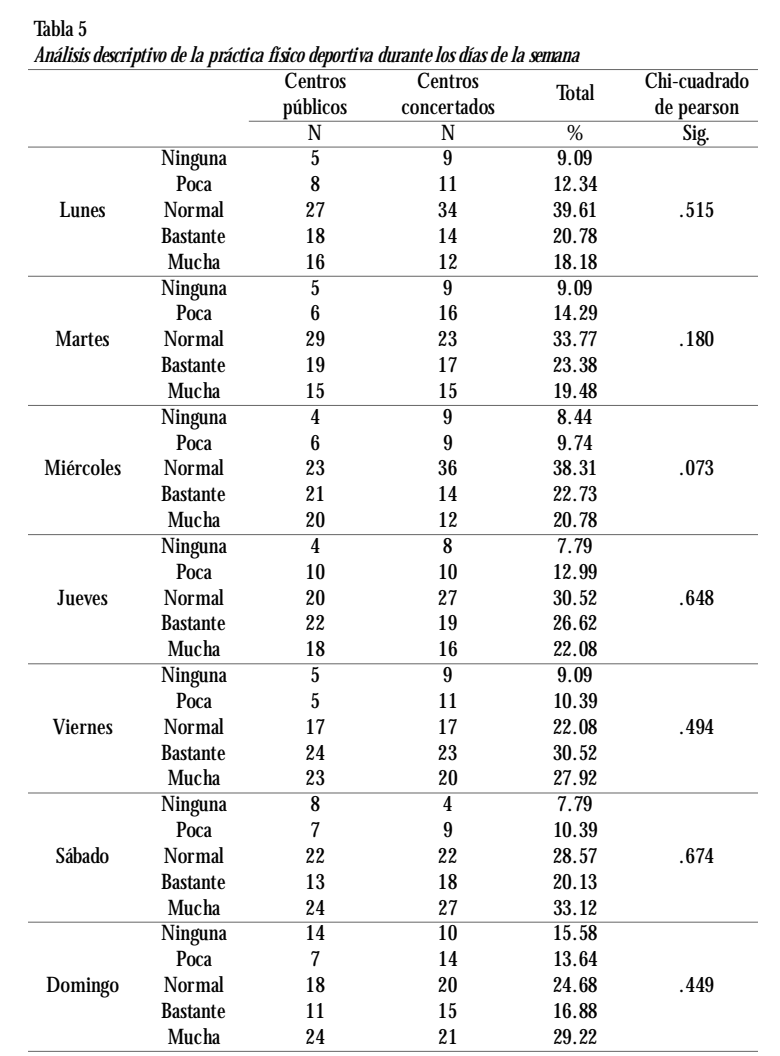

\section{Objetivo 4. Determinar la relación entre el en-} torno socioeconómico, los hábitos alimenticios y la actividad física que influyen en el estado nutricional de los estudiantes

En la tabla 6 se observa que existen diferencias significativas en función del tipo de centro. En esta tabla se puede comprobar la diferencia del estado de salud de los escolares, teniendo una gran influencia la economía familiar para una alimentación de calidad y variada. Se constatan diferencias significativas tras la fórmula del $\mathrm{CHI}^{2}$ entre las diferentes variables; alimentación, actividad física e IMC. Se muestra la diferencia en el estado de salud de los escolares entre un centro educativo y otro en ese periodo, haciendo referencia a los objetivos anteriores debido a las variables implicadas y obteniendo un resultado en este objetivo en el que los escolares de centros públicos sufrían más sobrepeso y obesidad, mientras que en los centros concertados el alumnado con normopeso era superior al de los centros públicos.

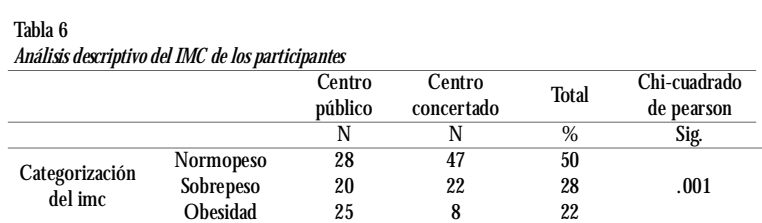

\section{Discusión}

El propósito de este estudio fue comparar la prevalencia de sobrepeso, obesidad y niveles de actividad física en relación con el estado socioeconómico de las familias de los diferentes centros educativos, con el fin de colaborar en el replanteamiento de estrategias y acciones que contribuyan a la disminución de esta problemá tica en las futuras generaciones.

Al realizar una comparativa de esta investigación con estudios relacionados, apunta a que la alimentación en los sujetos de los centros públicos analizados en edad escolar estaba lejos de ser la adecuada y la actividad física realizada no se acercaba a la ideal. Por lo que destaca la diferencia del estado de salud entre centros concertados y centros públicos, teniendo relación directa con el estado socioeconómico.

Los estudios con los que se han comparado los resultados se muestran en los siguientes apartados. El objetivo principal del presente estudio es conocer el estado de salud de los escolares relacionado con la actividad físico-deportiva, la alimentación y la desigualdad socioeconómica.

\section{Entorno socioeconómico del alumnado y los há- bitos alimenticios de los estudiantes}

En cuanto al entorno socioeconómico, diferentesautores como el estudio dirigido por Fairclough, Butcher \& Stratton (2008) con 193.628 escolares, los resultados mostraron que la participación es mayor en los centros públicos, siendo de 201.242 frente a 48.084 de los concertados; referidos a actividades que suponen un coste económico. Se tratan de unos val ores similares al presente trabajo, ya que la diferencia significativa que se encontró esque el alumnado de un centro público practicaba más variedad de actividad física que el escolar del centro concertado, por lo que la inversión económica en actividad física se inclina más en los escolares del centro público que del privado.

A continuación, se enfoca la discusión de los resulta dos relacionados con los hábitos alimenticios. Para Beltrán-Carrillo et al. (2017), en nuestro país disponemos de la dieta mediterránea como modelo referente de alimentación sana y saludable. Un modelo que si- 
guiendo los resultados del presente estudio no todo alumnado seguía, pero siguiendo a organismos como la SENC, que trabaja para que la sociedad ajuste su alimentación en base al modelo de Pirámide Alimentaria, que en la actualidad conocemos (Sociedad Española de Nutrición Comunitaria, 2017). En relación con la investigación, se comprueba como los estudiantes de los centros concertados consumían más lácteos que escola res del centro público, los lácteos están considerados un alimento relevante de la dieta mediterránea.

Por el contrario, en el estudio de Iglesias, del Pozo y López (2019) en relación a los al imentos ultra procesa dos se observó un elevado consumo, superando con creces el consumo ocasional y moderado que se aconseja, y rompiendo así todas las recomendaciones sobre una correcta alimentación. En la presente investigación, se observó cómo los alimentos ultraprocesados; dulces, zumos y comida rápida, sí eran consumidos por ambas escuelas educativas, rompiendo las recomendaciones en relación con el estudio citado anteriormente.

No parece extraño si tenemos en cuenta que los al imentos ultra procesados han aumentado considera blemente en nuestro día a día, llegando a triplicar su presencia en las listas de la compra (Latasa, Louzada, Steele, y Monteiro, 2017). El consumo de alimentos ultra procesados no solo resulta perjudicial por despla zar otros alimentos mucho más ricos, en cuanto a nutrientes se refiere, sino que también están relaciona dos con una mayor prevalencia de obesidad y sobrepeso en España (M endonça et al., 2016), con un mayor porcentaje graso (Costa, Del-Ponte, Assunção, y Santos, 2018).

En un reciente estudio (Inchley et al., 2017) en el que se analizó el consumo de alimentos por parte de adolescentes pertenecientes a 40 países europeos, España ocupaba el último lugar en el consumo tanto de vegetales como simultáneamente el consumo de frutas y vegetales. En la investigación realizada, se comprobó como el consumo de verduras era estable en el consumo de una vez al día, por el contrario, muy bajo en dos 0 más veces al día. Del mismo modo se situó el consumo de fruta, tomando la gran mayoría de estudiantes una vez al día y muy bajo dos o más veces al día.

\section{Analizar y comparar los niveles de actividad física de los participantes}

Análisis descriptivo del tipo de actividad física que practican los escolares en los diferentes centros educativos

González y Portolés (2014) obtuvieron resultados que corroboran los datos adquiridos en este estudio, ela borando un estudio en el que se reflejó que la mayoría de la muestra analizada realizaba actividad física y deportiva extraescolar de manera periódica añadiendo que deportes como el fútbol o el baloncesto son los más demandados, siendo también el fútbol o fútbol sala los deportes más demandados por los sujetos de este estudio.

Por otro lado, el O bservatorio del deporte de SeviIla (2007), obtiene en su estudio que los porcentajes de actividad física son muy similares en ambos tipos de centros, $70.72 \%$ en públicos y $72.86 \%$ en privadosconcertados; valores que no fueron similares a los adquiridos en el presente trabajo, ya que no se encontró diferencia significativa entre los diferentes centros.

Análisis descriptivo del tiempo que están activos los estudiantes en diferentes contextos y de la práctica físico deportiva durante los días de la semana

Según la Encuesta Nacional de Salud (M inisterio de Sanidad, Servicios Sociales e Igualdad, 2014), es a partir de los 9 años cuando este hábito de práctica de actividad física extracurricular comienza a tener especial relevancia, siendo los sujetos de esta investigación ma yores de 9 años. Cabe destacar la investigación realizada por Beltrán-Carrillo et al. (2017) en la que se manifiestan diferencias en el tiempo empleado en actividad sedentaria y actividad física en diferentes segmentos horarios del día, y en la que se hace hincapié en la necesidad de promover la actividad física. En relación a este estudio el mayor tiempo empleado en actividad física por ambos centros se encontró en horarios lectivos; " «activo en ef» y «activo en el recreo». Siendo el resto de momentos del día y de la semana, coincidiendo con horarios no lectivos, los que menos actividad física hacían.

LaAF practicada en los diferentes momentos del día presentaba diferencias entre el periodo de mañanas con el periodo de tardes, siendo el periodo de tardes el de menos actividad física (Greca et al., 2016), obteniendo así datos similares a la presente investigación donde se reafirmó que, en horario lectivo, los estudiantes practicaban mayor actividad física en horario de mañanas que en horario de tardes. 0 tros estudios recientes en Espa ña (BarjaFernández, Pino, Portela \& Leis, 2019; Zurita-O rtega, U bago-J iménez, Puertas-M olero, GonzálezValero, Castro-Sánchez \& Chacón-Cuberos, 2018) junto al estudio O BIN (Naranjo-O rellana et al., 2018) muestran datos similares con mayor frecuencia de actividad física en horario de mañanas que de tardes, siendo idénticos al presente trabajo. 
Para terminar, los hábitos de práctica de actividad física y la dieta sal udable en los jóvenes está influenciada por la familia pudiendo presentarse incluso como una barrera para la actividad física y una dieta saludable (Aguilar et al., 2014), por lo que cada vez más estudios incluyen la participación de las familias en sus intervenciones (Bibiloni et al., 2017). Diversos estudios han relacionado el nivel de actividad física de padres y madres con el de sus hijos, existiendo una mayor probabilidad de realizar actividad física en éstos si sus padres y ma dres son practicantes (Marques, González, Martins, Fernández-Villarino \& Carreiro, 2017).

Determinar la relación entre el entorno socioeconómico, los hábitos alimenticios y la actividad física que influyen en el estado nutricional de los estudiantes

En cuanto a la obesidad, diversos estudios realizados a nivel europeo (World Health O rganization, 2017) muestran cifras parecidas de sobrepeso y obesidad a las obtenidas en el presente estudio. Centrándonos en España, también encontramos estudios relacionados con la prevalencia de sobrepeso y obesidad con cifras similaresal nuestro (Antelo, Magdalena, y Reboredo, 2017). La evidenciarelacionada con España coincide con el presente estudio en afirmar que los adolescentes presentan niveles de sobrepeso y obesidad preocupantes (Bazán et al., 2017; Inchley et al., 2017), al igual que en el presente trabajo, ya que el $50 \%$ de los sujetos se encontra ban en sobrepeso y obesidad siendo la mayoría del centro público, mientras que en el colegio concertado la mayoría de escolares se encontraban en normopeso.

Siguiendo con losestudios en España, M eza, Sánchez, Bonilla y Jiménez (2016) revelan que en España el 13.9 $\%$ presenta problemas de obesidad, mientras que en el presente trabajo son el $22 \%$ de los menores que presentaban problemas de obesidad, si se combina este problema con el de sobrepeso, asciende hasta el $26.3 \%$ de la población estudiada, siendo el 50 \% entre sobrepeso y obesidad en la investigación desarrollada. De manera consecutiva se estableció un estudio estadístico sobre la obesidad, siendo tan solo 1 de cada 10 escolares que presentan obesidad (Pinel, Chacón, Castro, Espejo, Zurita y Pérez, 2017), en cambio en esta investigación, son 33 escolares, siendo 2 de cada 10 los estudiantes que se encontraban en estado de obesidad. Siguiendo a Martínez, Aznar \& Contreras (2015), los resultados de su estudio con 2.811 menores expresaron que la prevalencia de sobrepeso en escolares asciende al $13.7 \%$ y la obesidad al $14.5 \%$; valores inferiores en esta investiga ción para la preval encia de sobrepeso que fue de un 28
$\%$ y de obesidad que fue de un $22 \%$.

En la misma dirección, Sánchez-Echenique (2012), que llevó a cabo un estudio en la población española sobre una muestra de 978 discentes, obtuvo un $28.6 \%$ de sobrepeso en niños y en las niñas, el $23.5 \%$. Para la preval encia de obesidad, los niños fueron del $12.9 \%$ y las niñas $12.3 \%$; ; valores superiores al presente estudio para la prevalencia de sobrepeso total que fue de un $28 \%$ y de la obesidad de un $22 \%$. 0 tras investigaciones a destacar son las siguientes: López-Sobaler, RodríguezRodríguez, Ballesteros-Arribas, Pérez-Farinós y Ortega (2013) que desarrollaron un estudio en la Región de Murcia con un total de 178.894 menores, que mostra ron una prevalencia de sobrepeso del $25 \%$, para obesidad del $16.7 \%$; valores inferiores pero cercanos a los del presente estudio para la prevalencia en sobrepeso $28 \%$ y muy distantes de los datos obtenidos en la obesidad siendo en el presente trabajo del $22 \%$.

Por otro lado, el Ministerio de Sanidad, Servicios Sociales e Igualdad del año 2011/ 2012 obtuvo como resultados de una población de 10 a 14 años un porcentaje de obesidad del $3.2 \%$ y de prevalenciaen sobrepeso del $19.2 \%$; valores inferiores a los obtenidos en la investigación para la prevalencia en sobrepeso con el 28 $\%$ y obesidad con el $22 \%$.

Al contrastar estos resultados con los de estudios de otras regiones del mundo, se aprecia que esta problemática detectada en la niñez centroamericana es incluso más grave que en al gunas zonas. Por ejemplo, Mazidi et al., (2018), realizaron unarevisión sistemáticay meta análisis de estudios de prevalencia de sobrepeso y obesidad, realizados en niños y niñas (de 5 a 12 años de edad) y en adolescentes (de 12 a 19 años), de países de Asia, encontrando $5.8 \%$ de obesidad ( $7 \%$ en niños y $4.8 \%$ en niñas) y $11.2 \%$ de sobrepeso ( $11.7 \%$ en niños y $10.9 \%$ en niñas), siendo los resultados inferiores a la prevalencia encontrada en el presente estudio.

Anal izados estudios de otras partes del mundo, en la región centroamericana esta situación es particularmente un problema de salud pública, ya que la mayor cantidad de reportes de sobrepeso y obesidad en la niñez desde 1990 indican un ascenso de esta problemática en comparación con otras regiones (Cor valán et al. , 2017). La rapidez con la que han crecido los países de ingresos medios y bajos, han generado un mayor auge en la prevalencia del sobrepeso y obesidad, ocasionando un incremento en el desarrollo de diferentes padecimientos a muy temprana edad, tales como las enfermedades crónicas no transmisibles [ECNT], deficiencias en el crecimiento de los niños, retraso en el desarrollo mo- 
tor, problemas de desarrollo cognoscitivo e intelectual y trastornos psicosociales, los cuales pueden estar rela cionados con problemas de discriminación social, generando conductas agresivas, de exclusión social y aisla miento, que a su vez contribuyen al desarrollo de ansiedad, depresión, baja autoestima, entre otros trastornos (Aceves-Martins, Llauradó, Tarro, Solà \& Giralt, 2016).

Los hallazgos de este estudio indican que la niñez escolar centroamericana presenta una prevalencia de sobrepeso y obesidad de $24.9 \%$ y un $14.20 \%$ de bajo peso, los cuales dan evidencia del creciente problema de la mala nutrición como un fenómeno mundial, que afecta significativamente la salud de la población infantil (González et al., 2017). A poyado así por los resultados del presente estudio, ya que los sujetos con sobrepeso y obesidad fueron 1 de cada 2 en la totalidad. Estos resultados, pese a contrastar con estudios como los llevadosa cabo por Ramiro-González, Sanz-Barbero y RoyoBordonada (2017) o Díaz (2017), en los que sitúan la obesidad infantil como una problemática a nivel nacional de forma generalizada, se deben a que en estos colegios se había apostado de manera eficaz por la importancia de llevar a cabo una dieta equilibrada basada en el modelo de dieta mediterránea, elaborando planes internos para controlar tanto el hábito alimenticio en el horario escolar como fueradeél, motivando al alumnado para su consecución y logro y concienciando a toda la comunidad educativa sobre la problemática actual de obesidad infantil asociada a la ingesta de alimentos no saludables y el sedentarismo, objetos de estudio para esta investigación.

\section{Conclusiones}

Considerando los objetivos del estudio, se puede concluir que el estado de salud de las futuras generaciones estaba relacionado con los factores socioeconómicos influyendo directamente en la calidad de los alimentos que consumen, y no junto a la diversidad y cantidad de actividad física que realizan, ya que son los estudiantes del centro público los que realizan mayor variedad y cantidad de actividad física. La investigación sobre los factores socioeconómicos fue de gran importancia en el estudio para la interpretación del presente y futuro estado de salud del alumnado.

Analizar la situación socioeconómica de las familias era esencial para comprender y proponer cambios sociales que buscan una mayor estabilidad económica y el equilibrio entre zonascon un nivel socioeconómico bajo y alto. En esta corriente se encontraba la preocupación por el estado de salud de los escolares y las diferentes enfermedades respiratorias y cardiovasculares que puedan sufrir debido al sobrepeso y la obesidad.

En base a estas preocupaciones, se deben incorporar acciones de plan económico gubernamental, junto a las acciones desde la comunidad educativa donde se fomenten directamente hábitos de vida saludables, como son la actividad física y una dieta equilibrada y variada que elimine los alimentos ultraprocesados y bebidas azucaradas. Todo ello, con el fin de encaminar a la sociedad hacia la comprensión de métodos para una buena salud y calidad de vida.

\section{Limitaciones del estudio}

El presente estudio de investigación presentó una serie de limitaciones, principalmente de carácter económico, ya que el trabajo no derivó de un proyecto de investigación y no tuvo financiación al guna. Exististieron además limitaciones temporales, debido a que se tuvo que contar con un tiempo reducido para el desarrollo del estudio, contando con el tiempo cedido de la Escuela de Enfermería de Cartagena para obtener el instrumento de medida del peso y la altura. El tiempo de diferentes miembros de la comunidad educativa, como es el profesorado que cede parte de sus horas de clase para que los escolares puedan asistir a la toma de datos, registro de datos que se extræen de los cuestionarios y la extracción de datos sobre el peso y la altura de los estudiantes en los diferentes colegios de la Región de Murcia, más la accesibilidad de los objetos materiales de estudio.

Los resultados pretenden contribuir a la comprensión del sobrepeso y obesidad infantil, aunque entre en sus limitaciones se encontraba el número de participantes, por la dificultad de acceder a un número mayor de participantas derivadas de las limitaciones destacadas anteriormente. Aun así, este trabajo pretende sumarse al resto de estudios para contrarrestar la obesidad y el sobrepeso infantil y promover el bienestar integral.

\section{Implicaciones prácticas derivadas de los hallazgos del estudio}

Los resultados del presente estudio se enfocan hacia la necesidad de realizar proyecciones y charlas en las aulas con el fin de sensibilizar a la comunidad educativa. Considerándose una aplicación socioeducativa y de mejora para promover una serie de mejoras con la finali- 
dad de enfrentar el problema de salud que la mayoría de la sociedad sufre.

La finalidad es que los estudiantes sean conscientes de la importancia que la actividad física, acompañada de una alimentación saludable, tiene en el estado de salud.

Para finalizar, las implicaciones prácticas de este estudio derivan hacia la siguiente pregunta; ¿Se considera que los estudiantes de Educación Primaria tienen las mismas posibilidades de mantener una actividad física y alimentación para conseguir un estado saludable? Así, acaba la aplicación socioeducativa y de mejora realizada en este estudio para que las autoridades políticas y educativas capten el mensaje que se ha querido transmitir con la importancia que cada vez tiene más relevancia para realizar actividad física y mantener una buena al imentación, como es la capacidad económica de las fa milias.

\section{Referencias}

Aceves-Martins, M., Llauradó, E., Tarro, L., Solà, R., \& Giralt, M. (2016). Obesity-promoting factors in Mexican children and adolescents:Challengesand opportunities. Global HealthAction, 9(1). https:/ / doi.org/ 10.3402/ ghav9.29625

Afshin,A., Sur, P.J., Fay, K.A., Cornaby, L., Ferrara, G., Salama,. .S. \& Murray, C. J. (2019). Health effects of dietary risks in 195 countries, 1990-2017: a systematicanalysisfor theGlobal Burden of DiseaseStudy 2017. Lancet, 393(10184), 1958-1972. https:/ / doi.org/ 10.1016/ S01406736(19)300418

Aguilar, M.J., O rtegón, A., Mur, N., Sánchez, J. C., García,.J. ., García, I., \& Sánchez,A. M. (2014). Programasdeactividad físca para reducir sobrepeso y obesidad en niños y adolescentes revisión sistemática. Nutrición Hospitalaria, 30(4), 727-740.

AjejaBazán, M. J., Sellán Soto, M. D. C., VázquezSellán,A., Díaz Martínez, M. L., \& Domínguez Fernández, S. (2018). Factors associated with overweight and childhood obesity in Spain according to the latest national health survey (2011). Escola Anna Nery, 22(2).

Alba-Martín, R. (2016). Prevalencia de obesidad infantil y hábitos alimentariosen educación primaria. Enfermería Global, 15(2), 4062.

Antelo, M., Magdalena, P., y Reboredo,J.C. (2017). Obesity'A major problem for Spanish minors. Economicsand human biology, 24, 61-73. Doi: 10.1016/ j.ehb.2016.11.002

Atanasova, D. \& Koteyko, N. (2017). Obesity framesand counterframesin British and German onlinenewspapers. $H$ ealth, 21(6), 650-669.

Bagherniya, M., Sharma, M., Mostafai Darani, F., Maracy, M. R., Safarian, M.,Allipour Birgani, R., Keshavarz,S.A. (2017). Schoolbased nutrition education intervention using social cognitivetheory for overweight and obeseiranian adolescent girls.A Cluster randomized controlled trial. International Quarterly of Community H ealth Education, 38(1), 37-45. https:/ / doi. org/ 10.1177/ $0272684 X 17749566$

BajazFernández,S. Pino, M., Portela, I., \& Leis, R. (2019). Evaluación de los hábitos de alimentación y actividad física en escolares gallegos. Nutrición Hospitalaria, 1-26. http:/ / dx. doi.org/ 10.20960/ nh.02668

Bazán, M.J.A., Trujillo, M. I.J.,W ärnberg, J., Fernández, S. D., de Andrés,A. L., yFarinós, N. P. (2017). Differencesin theprevalence of diagnosisof overweight-obesity in Spanish children according tothe diagnosic criteria set used. Gaceta sanitaria. DO I: 10.1016/ j.gaceta 2017.07.014

Beltrán-Carrillo,V.J., Sierra, A. C., Jiménez Loais, A., GonzálezCutre, D., Martínez Galindo, C. yCervelló, E. (2017). Dife rencias según género en el tiempo empleado por adolescentesen actividad sedentaria y actividad físca en diferentes segmentos horarios del día. Retos. NuevastendenciasenEducaciónFísica, Deportey Recreación, (31).

Bibiloni, M. M., Fernández-Blanco, . ., Pujol-Plana, N., Surià, S., Pujol-Puyané, M. C., Mercadé, S., \&Tur, J.A. (2017). Reversión desobrepeso y obesidad en población infantil del/lafranca del Penedès: ProgramaACTIVA'T (2012). GacetaSanitaria. https:/ / doi.org/ 10.1016/ j.gaceta.2017.10.002

Brettschneider, A. K., Schienkiewitz,A., Schmidt, S., Ellert, U., \& Kurth, B. M. (2017). U pdated prevalencerates of overweight and obesity in 4-to 10-year-old children in Germany. Results from the telephone based KiGGSWave 1 after correction for biasin parental reports. European journal of pediatrics, 176(4), 547-551.

Campos, F., González-Víllora, S., Gómez, D. G., \& Martins, F.M. L. (2019). Benefits of 8-week fitness programs on health and fitness parameters (Beneficiosdelosprogramasdefitnessdeocho semanasen parámerosdesaludy fitness). Retos(35), 224-228.

Chacón, R.,Arufe,V., Espejo,T., Chacón,J.,ZZurita, F.y Castro, D. (2017). Práctica físico-deportiva, actividades de ocio y conceoción sobrela Educación Físca en escolares deA Coruña. Revista Retos. Nuevastendencias en Educación Física, Deportey Recrea ción, 32, 163-166.

Cigarroa, I., Sarqui, C. , \& Zapđatamana, R. (2016). Efectosdel sedentarismo y obesidad en el desarrollo psicomotor en niños y niñas: Una revisión dela actualidad latinoamericana. U niversidad y Sa lud, 18(1), 156-169. https:/ / doi.org/ 10.22267/ rus. 161801.27

Clark, H., Coll-Seck,A. M., Banerjee,A., Peterson, S., Dalglish,S. L.,Ameratunga, S. , Clæeson, M. (2020). A futurefor theworld's children? A WH O - UN ICEF- Lancet Commission. The Lancet, 395(10224), 605-658. https:/ / doi.org/ 10.1016/ S01406736(19)32540-1

Corvalán, C., Garmendia, M. L., Jones-Smith, J., Lutter, C. K., Miranda, J.J., Pedraza, L. S., Popkin, B. M., Ramiréz-Zea, M., Salvo, D. \& Stein, A. D. (2017). Nutrition status of children in Latin America. O besity Reviews, 18(July), 7-18. https:/ / doi.org/ 10.1111/ obr.12571 
Costa, C. S., Del-Ponte, B., Assunção, M. C. F., ySantos, I S. (2018). Consumption of ultra-processed foodsand body fat during childhood and adolescence a systematicreview. Publichealthnutrition, 21(1), 148-159. Doi: 10.1017/ S1368980017001331

Deckers, K.,Van Boxtel, M.,Verhey, F. \& Köhler, S. (2017). Obesty and cognitivededinein adults, effect of methodological choicesand confounding by agein a longitudinal sudy. Journal of Nutrition Heath andAging, 21(5), 546-553.

Delgado-Floody, P., Carter-Thuillier, B., Jerez-Mayorga, D., Cofré Lizama,A., \&M artínez-Salazar, C. (2018). Relación entresobrepeso, obesidad y niveles de autoestima en escolares (Relation between overweight, obesity, and self-eteem leves sin school children). Retos, $0(35), 67-70$.

Dízz, J. (2017). Childhood obesity: :Prevention or treatment?A nales de Pediaría, 86(4), 173-175.

Doepke, M ., \& Zilibotti, F. (2019). Love, money, and parenting: How economicsexplainstheway weraiseour kids. Princeton U niversity Press.

Esteves, D.,Vieira, S., Brás, R., O'Hara, K., \& Pinheiro, P. (2017). Nivel deatividadefísica ehábitos devida saudável de universitários portuguesss. RevistalberoamericanadePsicologíadel Ejercicio yel Deporte, 12(2), 261-270.

Fairclough,S. J., Butcher, Z. H., \& Stratton, G. (2008) Primary school children'shealth-enhancing physical activity patterns.theschool asa significant environment? Education; 3-13, 36(4), 371-381.

González,J . y Portolés, A. (2014). Actividad física extraescolar: relacionescon la motivación educativa, rendimiento académico y conductasasociadasa la salud. Revista IberoamericanadePsicología del Ejercicio y el Deporte, 9(1), 51-65.

González, G. ,Zurita, F., Puertas, P., Espejo,T., Chacón, R., \& Cas tro, M. (2017). Influencia de los factores sedentarios (dieta y videojuegos) sobre la obesidad en escolares de Educación Primaria. Reidocrea, 6(11), 120-129. Recuperado dehttp:/ / hall.net/ 10481/ 45233

Greca, J. P. deA., Silva, D. A. S., \& Loch, M. R. (2016). Physical activity and screen timein children and adolescentsin a medium size town in the South of Brazil. RevistaPaulistadePediatria, 34(3), 316-322. https:/ / doi.org/ 10.1016/ j.rppede.2016.01.001

Guillamón,A. R., Canto, E. G., y López, P.J. C. (2019). Reación entrecapacidad aeróbica y e nivel deatención en escolaresdeprimaria (Relationship between aerobic capacity and level of attention in primary school children). Retos, (35), 36-41.

Iglesias, Á., del Pozo, E. M. P., \& López,J. M. (2019). Prevalencia de sobrepeso y obesidad, hábitosal imentariosy actividad física y su relación sobreel rendimientoacadémico. Retos: nuevastendenciasen educaciónfísica, deportey recreación, (36), 167-173.

Inchley, J., Currie, D., Jewell, J., Breda, J., \& Barnekow,V. (2007). Adolescent 0 besity and Related Behaviours.Trendsand Inequalitiesin theW H O European Region, 2002-2014. O bservationsfrom the Health Behaviour in School-Aged Children (HBSC)W HO CollaborativeC ross-N ationa Study.

Knox, E., yM uros, J.J. (2017). Association of lifestylebehaviourswith self-eteem through health-redated qual ity of lifein Spanish adol escents European journal of pediatrics, 176(5), 621-628. D oi: 10.1007/ s00431-017-2886-Z

Latasa, P., Louzada, M. L. D. C., Steele, E. M., y M onteiro, C.A. (2017). Added sugarsand ultra processed foodsin Spanish households (1990-2010). European journal of clinical nutrition, 72(10), 1404-1412. Doi: 10.1038/ s41430-017-0039-0

Lđtorre, P., M ora, D., \& García, F. (2016). Prácicasdealimentación, actividad física y condición física de niños preescolares españoles Influencia devariablessociodemográficas. ArchivosArgentinosde Pediatría, 114(5), 441- 447. http:/ / dx.doi.org/ 10.5546/ aap. 2016.441

O rtegaAnta, R. M., López-Sobaler,A. M., \& Pérez-Farinós, N. (2013). Factoresasociadosal padecimiento deobesidad en muestras representativasdela población eppañola. Nutrición Hospitalaria, 28, 56-62.

Martínez, J.,Aznar,S., \& Contreras, O. (2015). El recreo escolar como oportunidad deespacio y tiempo saludable. RevistaInternacional deM edicinay Ciencias delaActividad Físicay del Deporte/ International Journal ofM edicineand Scienceof PhysicaActivity and Sport, 15(59), 419-432.

Martínez, C. P., Cuberos, R. C., Sánchez, M. C., Garcés, T. E., Ortega, F.Z., \& Cortés,A.J. P. (2017). Diferenciasdegénero en relación con el ÍndicedeM asa Corporal, calidad dela dieta y actividadessedentariasen niñosde 10 a 12 años. Retos. nuevastendenciasen educaciónfísica, deportey recreación, (31), 176-180. Recuperado de https:/ / dialnet. unirioja. es/ servlet/ articulo?codigo $=5841367$

Marques, A., González, M., M artins, J., Fernández-Villarino, M. A. \& \& Carreiro, F. (2017). Relación entrela actividad física delos adolescentes y la de madres/ padres. Revista de Psicología del Deporte, 26(1), 145-156.

Mazidi, M., Banach, M., Pascal-Kengne, A., \& Lipid and Blood Pressure M eta-analysis Collaboration Group. (2018). Prevalence of childhood and adolescent overweight and obesty in Asian countries a systematic review and meta-analysis. Arch M ed Sci, 14(6), 1185-1203. https:/ / doi. org/ 10.5114/ aoms. 2018.79001

Mendonça, R. D. D., Pimenta,A.M.,Gea,A., delaFuenteArrillaga, C., Martinez-Gonzalez, M.A., Lopes,A. C. S., yBes-Rastrollo, M. (2016). Ultraprocessed food consumption and risk of overweight and obesity: the Universty of N avarra Follow-U P (SUN ) cohortsudy, 2. TheAmerican journal of clinical nutrition, 104(5), 14331440. Doi: 10.3945/ àcn.116.135004

Meza, R., Sánchez, F., Bonilla, R yj iménez, C., (2016). Prevalencia de sobrepeso y obesidad en niños de Escuelas de Santo D omingo de Heredia, CostaRica RevHisp CiencSalud. 2(1), 12-20.

M inisterio de Sanidad, ServiciosSocialese lgualdad (2014). Encuesta N acional de Salud. España 2011/ 12. Actividad física, des cansoy ocio. Serieinformesmonográficos, 4.

Naranjo-O rellana, J.,Alonso-Alfonseca, F.J., Carranza-Márquez, M.D., \& RuedaPuente, J. D. (2018). Hábitosnutricionalesy de 
actividad física en escolaresdeeducación primaria: datosdel estudio longitudinal «OBIN » 2011-2017. RevistaAndaluzadeM edicinadel Deporte, 11(4), 199-204. Recuperado de: https:/ / dialnet. unirioja es/ servlet/ articulo?codigo $=6841611$

deSevilla, O. D. D. (2007). Hábitosy actitudesdelossevillanosmayores anteel deporte. Sevilla: Instituto M unicipal deD eportes,Ayuntamiento deSevilla. O MS. Recomendacionesmundialessobreactividad físicaparalasalud. O rganización M undia dela Salud. Ginebra. 2010. Disponible en: http:// whqlibdoc. who.int/ publications/2010/ 9789243599977 spa.pdf?ua=1

OMS. O besidady Sobrepeso. Ginebra, 2012. Disponibleen: http:/ / www. who.int/ mediacentre/ factsheets/ fs311/ es/

O rganizaciónM undia delaSalud. (2018). Actividadfísca. Datosy cifras. Recuperado de: http:/ / www.who. int/ es/ newsroom/ fact-sheets/ detail/ physical-activity

Palomino-Devia, C., O tero-Saborido, F. M., \& González-Jurado,J. A. (2016). Análisisdela adi posidady la condición física en escolares colombianos. Biomédica, 36(3), 343-353.

Pinel, C., Chacón, R., Castro, M., Espejo,T.,Zurita, F. yCortés,A. (2017). Diferencias de género en relación con el Índice de Masa Corporal, calidad dela dieta y actividadessedentariasen niñosde10 a 12 años. Retos, 31, 176-180

Poitras,V.J., Gray, C.E., Borghese, M. M., Carson,V., Chaput, J.-P., Janssen, I. yTremblay, M. S. (2016). Systematic review of the relationshipsbetween objectively measured physical adivity and health indicatorsin school-aged children and youth. A pplied Physiology, Nutrition, and Metabolism = PhysiologieA ppliquee, Nutrition EtM etabolisme, 41(6Suppl 3), S197-239. https:/ / doi.org/ 10.1139/ apnm-2015-0663

Sánchez-Echenique, M. (2012). Aspectosepidemiológicosdela obesidadinfantil. PediatríaAtención Primaria, 14, 9-14.

Ramiro-González, M., Sanz-Barbero, B. y Royo-Bordonada, M. (2017). Childhood excess weight in Spain from 2006 to 2012. Determinantsand parental misperception. RevistaEspañola deCardiología, 70(8), 656-663.

Ramos, P., Jiménez-Iglesias, A., Rivera, F., \& M oreno, C. (2016). Evolución de la práctica de la actividad físca en los adolescentes españoles. Revistalnternacional de M edicinay Cienciasdela Actividad Físicay del Deporte, 16(62), 335-353.

Ríos, C. (2019). Comecomida real: una guía para transformar tualimentación y tu salud. Paidós.

Rosa,A.,Carrillo, P.J., Garća, E., Pérez,J...,,Tarraga, L., \&Tarraga, P. J. (2019). Dieta mediterránea, estado de peso y actividad física en escolares de la Región de Murcia. Clinica E. Investigación en Arteriosclerosis, 31(1), 1-7.DO I: https:/ / doi.org/ 10.1016/ j.arteri.2018.09.002

Rosa-Guillamón,A, GarcíaCantó,E., \&Carrillo-López, P.). (2018). La educación físca como programa dedesarrollofíśco y motor. Emás: revistadigital deeducaciónfísica, (52), 105-124.

Sanz, D. (2017). N ivelesdeactividad física moderada-vigorosa deadolescentesdel municipio deSoria. SportisSciJ, 3(1), 100-122.
Saunders,T.J. . \&Vallance, J. K. (2017). Screen timeand health indicators among children and youth: current evidence, limitationsand future directions Applied heattheconomicsand heath policy, 15(3), 323-331.

Sevil, J., Abós, Á., Generelo, E. , Aibar,A ., \& García-González, L. (2016). Importancia del apoyo a lasnecesidadespsicológicasbáscas en la predisposición hacia diferentes contenidosen Educación Física. Retos N uevastendenciasenEducaciónFísica, Deportey Recreación, 29, 3-8.

Sociedad Española de Nutrición Comunitaria (2017). Nutricióncomunitaria: PiramideAlimentacion SaludableSEN C-Edad escolar. Recuperado de: http:/ / www. nutricioncomunitaria org/ es/ noticia/ sepresentanlasnuevas-guias-alimentarias-parala-poblacionespanola elabora das-por-la-senc-con-la

Son, W. M., Sung, K. D., Bharah, L. P., Choi, K. J., y Park, S.Y. (2017). Combined exercisetraining reducesblood pressure, arterial siffness, and insulin resistance in obese prehypertensive adolescent girls. Clinical and Experimental Hypertension, 39(6), 546552. Doi: 10.1080/ 10641963.2017.1288742

Taylor,.J. H., Xu,Y., Li, F., Shav, M., Dziura, J., Caprio, S. Savoye, M. (2017). Psychosocial predictorsand moderators of weight management programme outcomes in ethnically diverse obese youth. Pediatric O besity, 12(6), 453-461. https:/ / doi.org/ 10.1111/ ijpo. 12165

van Hooft, J., Patterson, C., Löf, M.,Alexandrou, C., Hilton, S., \& Nimegeer,A. (2018). M edia framingand construction of childhood obesity: a content analysis of $S$ wedish newspapers. O besity science \& practice, 4(1), 4-13.

Vasquez, F., Diaz, E., Lera,L., Meza,J.,Curi, K.,Torres,].,\&Burrows, R. (2017). Physical fitnessand insul in sensitivity in a group of obese children from 8 to 13 years of ageby puberal sate. NUTRICIO N HOSPITALARIA, 34(4), 808-813. https:/ / doi. org/ 10.20960/ nh.61

W HO (2016). O besity and Overweight. Retrieved J uly 5, 2018, from http:// www. who.int/ news-room/ fact-sheets/ detail/ obesity-andoverweight

World HealthO rganization. (2017). Tenfold increasein childhood and adolescent obesity in four decades new study by Imperial College London andW H O. Saudi M edical Journa, 38(11), 1162-1163.

Zurita, F., Castro, M., Linares, M., Chacón, R. (2017). Resiliencia, un elemento deprevención en actividadfísica. Sportis, 3(1), 50-62

Zurita-O rtega, F., U bago-Jiménez, J. L., Puertas-M olero, P., González-Valero, G., Castro-Sánchez, M ., \& Chacón-Cuberos, R. (2018). N ivelesdeactividad físca en alumnado deEducación Primaria dela provincia deGranada. Retos, (34), 218-221. Recuperado de: https./ / recyt. fecyt.es/ index. php/ retos/ article/ view/ 60098/ 38735 